\title{
GROUP STRATEGYPROOFNESS IN QUEUEING MODELS
}

\author{
MANIPUSHPAK MITRA AND SURESH MUTUSWAMI
}

\begin{abstract}
We examine the tradeoffs between two variants of group strategyproofness, efficiency and budget balance in queueing models. In general, group strategyproofness is incompatible with efficiency and budget balance. Weakening budget balance to feasibility, we show that the incompatibility persists with strong group strategyproofness. We then identify a necessary condition for weak group strategyproofness and efficiency and use it to show that these two requirements are incompatible with budget balance unless there are exactly three agents. We also demonstrate the compatibility when there are three agents. Finally, we identify a class of efficient and weak group strategyproof mechanisms that we call $k$-pivotal mechanisms and identify the complete subclass of these mechanisms that are feasible.
\end{abstract}

\section{INTRODUCTION}

In a queueing environment, agents wish to avail of a service provided by one or more servers, each of whom can serve the agents only sequentially. Agents incur idiosyncratic waiting costs. Maniquet [5] notes that queueing models capture many economic environments. Such models have been examined from both incentive and axiomatic viewpoints in a recent series of papers.

The problem from an incentive viewpoint stems from the fact that the information needed to ensure that agents are served efficiently - that is, the queue should minimize the aggregate waiting cost - is not known publicly. This information has to be elicited from the agents by providing suitable incentives in the form of transfers. As long as we restrict our attention to a single server problem, it is possible to achieve the first best (truth-telling in dominant strategies, efficiency and budget balance) if waiting costs are a linear function of time. ${ }^{1}$ In more complex setups - for instance, when there are multiple servers - the first best is typically difficult to achieve. ${ }^{2}$

Even when the first best is achievable, the mechanisms that are obtained are vulnerable on various counts. For instance, since truth-telling is not a

Date: April 5, 2006.

${ }^{1}$ See Mitra [6], [7] and Suijs [12].

${ }^{2}$ See Mitra [8]. 
unique dominant strategy, the direct mechanism has multiple Nash equilibria and one can construct examples to show that there are Nash equilibria that are inefficient. The mechanisms are also vulnerable to manipulations by groups of agents. The objective of this paper is to examine the tradeoffs between stronger notions of non-manipulability, efficiency and budget balance.

We use two variants of the notion of immunity to manipulation by coalitions. ${ }^{3}$ Strong group strategyproofness requires that there does not exist a deviation which makes all deviating members weakly better off and at least one member strictly better off. In contrast, weak group strategyproofness only requires that there does not exist a deviation which makes all deviating members strictly better off.

We show that both variants of group strategyproofness are, in general, incompatible with efficiency and budget balance. We therefore, weaken budget balance to feasibility whereby the mechanism is allowed to run a surplus but not a deficit. While strong group strategyproofness is incompatible even with efficiency and feasibility, weak group strategyproofness yields more positive results. We identify a necessary condition for weak group strategyproofness and efficiency and use it to show that these two requirements are incompatible with budget balance when there are four or more agents. We also show that these requirements are incompatible with two agents; however, they are compatible when there are three agents. Next, we identify a sufficient condition for weak group strategyproofness and efficiency. We use this condition to identify a class of mechanisms we call $k$-pivotal mechanisms and show that if a mechanism in this class is efficient, then it is weak group strategyproof. Finally, we identify the subclass of $k$-pivotal mechanisms which are also feasible.

In what follows, we setup the model in Section 2 and derive the results in Section 3. We conclude in Section 4.

\section{MOdeL}

Let $N,|N| \geq 2$ be the set of agents. Each agent wants to consume a service provided by a server. Agents can be served only sequentially and serving any agent takes one unit of time. Each agent is identified with a waiting $\operatorname{cost} \theta_{i} \in \Re_{+}$, her cost of waiting per unit of time. The waiting cost of an agent is known only to that agent. A profile $\theta=\left(\theta_{i}\right)_{i \in N}$ is a vector of waiting costs of all agents. The profiles $\theta$ and $\theta^{\prime}$ are $S$-profiles if $\theta_{i}=\theta_{i}^{\prime}$ for all $i \in N \backslash S$. Given a profile $\theta$ and $S \subsetneq N$, the profile $\left(\theta_{j}\right)_{j \notin S}$ will usually be

${ }^{3}$ See also Bogomolnaia and Moulin [1], Moulin and Shenker [10] and Mutuswami [11]. 
denoted $\theta_{-S}$. Sometimes, though, we will find it convenient to enumerate the elements of $S$ and we will adopt the notations $\theta_{-i}, \theta_{-i-j}$ and so on.

A queue is a bijection $\sigma: N \rightarrow\{1, \ldots,|N|\}$. For notational convenience, we will denote $\sigma(i)$ as $\sigma_{i}$ for all $i \in N$. If $\sigma_{i}=k$-that is, agent $i$ occupies position $k$ in the queue - then her waiting cost is $(k-1) \theta_{i}$. Let $\Sigma(N)$ be the set of all queues of agents in $N$. For every $i \in N$ and $\sigma \in \Sigma(N), P_{i}(\sigma)=$ $\left\{j \in N \mid \sigma_{j}<\sigma_{i}\right\}$ is the set of predecessors of $i$ while $P_{i}^{\prime}(\sigma)=N \backslash\left(P_{i}(\sigma) \cup\{i\}\right)$ is the set of successors. A queue $\sigma^{*}$ is efficient for the profile $\theta$ if it minimizes the aggregate waiting cost: $\sigma^{*}=\operatorname{argmin}_{\sigma \in \Sigma(N)} \sum_{i \in N}\left(\sigma_{i}-1\right) \theta_{i}$. We denote the set of efficient queues for given $N$ and $\theta$ as $\Sigma^{*}(N, \theta)$.

A mechanism $\mu$ associates to each profile $\theta$, a tuple $(\sigma(\theta), t(\theta)) \in \Sigma(N) \times$ $\Re^{N}$. Agent $i$ 's outcome is denoted as $\left(\sigma_{i}(\theta), t_{i}(\theta)\right)$ where $\sigma_{i}(\theta)$ is the queue position and $t_{i}(\theta)$ the corresponding transfer. Let $u_{i}\left(\sigma_{i}(\theta), t_{i}(\theta), \theta_{i}^{\prime}\right)=-\left(\sigma_{i}(\theta)-\right.$ 1) $\theta_{i}^{\prime}+t_{i}(\theta)$ denote $i$ 's utility when the profile $\theta$ is reported (collectively) and her true waiting cost is $\theta_{i}^{\prime}$.

Definition 1. A mechanism $(\sigma, t)$ is strategyproof if for all $i \in N$ and all $i$-profiles $\theta, \theta^{\prime}, u_{i}\left(\sigma_{i}(\theta), t_{i}(\theta), \theta_{i}\right) \geq u_{i}\left(\sigma_{i}\left(\theta^{\prime}\right), t_{i}\left(\theta^{\prime}\right), \theta_{i}\right)$.

Strategyproofness implies that truth-telling is an optimal strategy for every agent irrespective of the announcements of other agents. Two ways of strengthening the notion of strategyproofness to coalitions are as follows.

Definition 2. A mechanism $(\sigma, t)$ is strong group strategyproof if for all $S \subset N$ and all $S$-profiles $\theta, \theta^{\prime}, u_{i}\left(\sigma_{i}(\theta), t_{i}(\theta), \theta_{i}\right) \leq u_{i}\left(\sigma_{i}\left(\theta^{\prime}\right), t_{i}\left(\theta^{\prime}\right), \theta_{i}\right)$ for all $i \in S$ implies $u_{i}\left(\sigma_{i}(\theta), t_{i}(\theta), \theta_{i}\right)=u_{i}\left(\sigma_{i}\left(\theta^{\prime}\right), t_{i}\left(\theta^{\prime}\right), \theta_{i}\right)$ for all $i \in S$.

Definition 3. A mechanism $(\sigma, t)$ is weak group strategyproof if for all $S \subset$ $N$ and all $S$-profiles $\theta, \theta^{\prime}, u_{i}\left(\sigma_{i}(\theta), t_{i}(\theta), \theta_{i}\right) \geq u_{i}\left(\sigma_{i}\left(\theta^{\prime}\right), t_{i}\left(\theta^{\prime}\right), \theta_{i}\right)$ for at least one $i \in S$.

Strong group strategyproofness rules out all deviations by groups of agents which leave all deviating agents weakly better off and at least one deviating agent strictly better off while weak group strategyproofness only rules out misreporting that strictly improves the utility of each member of the deviating coalition.

Definition 4. A mechanism $(\sigma, t)$ is efficient if for all profiles $\theta, \sigma(\theta) \in$ $\Sigma^{*}(N, \theta)$.

It is easy to see that efficiency implies that if $\theta_{i}>\theta_{j}$ then $\sigma_{i}<\sigma_{j}$. However, the efficient queue may not unique: if, for example, all agents announce the same waiting cost then all queues are efficient. In this paper, we only consider an efficient rule which is a single valued selection from the 
efficiency correspondence. This is done by selecting an arbitrary order $\succ$ on the set of agents $N$ and using the following tie breaking rule: if $i \succ j$ and $\theta_{i}=\theta_{j}$ then $\sigma_{i}<\sigma_{j}$. This tie-breaking rule is also applied for all queueing situations involving a subset $S$ of the agents. In what follows, we will sometimes use $\sigma^{*}(\theta)$ to denote the efficient queue consistent with the above tie-breaking rule for the profile $\theta$.

Definition 5. A mechanism $(\sigma, t)$ is budget balanced if for all profiles $\theta$, $\sum_{i=1}^{n} t_{i}(\theta)=0$. The mechanism is feasible if $\sum_{i=1}^{n} t_{i}(\theta) \leq 0$.

We end this section with a bit of notation. Given $x \in \Re$, we use $N(x, \epsilon)$ to denote the $\epsilon$-neighbourhood of $x$ in the usual topology.

\section{Results}

The following is a well-known characterization of strategyproof and efficient mechanism when preferences are quasi-linear.

Lemma 1. An efficient mechanism $(\sigma, t)$ is strategyproof if and only if

$$
t_{i}(\theta)=-\sum_{j \neq i}\left(\sigma_{j}(\theta)-1\right) \theta_{j}+g_{i}\left(\theta_{-i}\right) \text { for all } i \in N .
$$

Proof: Follows from Theorem 1 of Holmström [4] since the domain of profiles $\Re_{+}^{N}$ is convex.

Remark 1. The efficient mechanism with the transfers satisfying (1) is known as the VCG mechanism. ${ }^{4}$ The function $g_{i}\left(\theta_{-i}\right)$ cannot depend on either $\theta_{i}$ or $\sigma_{i}(\theta)$ but it can depend on the queue positions of agents in the economy where agent $i$ is not present.

Remark 2. The pivotal mechanism ${ }^{5}$ is the efficient mechanism for which the functions $g_{i}$ are given by

$$
g_{i}\left(\theta_{-i}\right)=\sum_{j \neq i}\left(\sigma_{j}^{*}\left(\theta_{-i}\right)-1\right) \theta_{j} \text { for all } i \in N .
$$

It follows from Lemma 1 that this mechanism is strategyproof. It can also be verified that (2) can be written as

$$
t_{i}(\theta)=-\sum_{j \in P_{i}^{\prime}(\sigma(\theta))} \theta_{j} \text { for all } i \in N .
$$

Lemma 2. An efficient mechanism $(\sigma, t)$ is strategyproof if and only if

$$
t_{i}(\theta)=-\sum_{j \in P_{i}^{\prime}(\sigma(\theta))} \theta_{j}+h_{i}\left(\theta_{-i}\right) \text { for all } i \in N .
$$

\footnotetext{
${ }^{4}$ See Vickrey [13], Clarke [2] and Groves [3].

${ }^{5}$ See Clarke [2] and Moulin [9].
} 
Proof: Without loss of generality, substitute $g_{i}\left(\theta_{-i}\right)=\sum_{j \neq i}\left(\sigma_{j}^{*}\left(\theta_{-i}\right)-\right.$ 1) $\theta_{j}+h_{i}\left(\theta_{-i}\right)$ in Lemma 1 . The result then follows immediately.

We now move to our main results. In proving these results, we will assume, without loss of generality, that the set of agents is given by $N=\{1, \ldots, n\}$. Our first result shows that there are no efficient mechanisms which are strong group strategyproof and feasible.

Theorem 1. There exists no efficient mechanism satisfying strong group strategyproofness and feasibility.

Proof: Let $(\sigma, t)$ be an efficient mechanism satisfying strong group strategyproofness and feasibility. Let $\theta$ be a profile such that $\theta_{1}>\ldots>\theta_{n}$ and let $\delta=\sum_{i=1}^{n} t_{i}(\theta)$. Efficiency implies that $\sigma_{i}(\theta)=i$ and feasibility that $\delta \leq 0$. Choose $\delta^{\prime}>-\delta$ and let $\theta^{\prime}$ be a $\{1,2\}$-profile of $\theta$ such that $\theta_{i}^{\prime}=\theta_{i}+\delta^{\prime}, i=1,2$. By efficiency, $\sigma\left(\theta^{\prime}\right)=\sigma(\theta)$. We show that $t_{i}(\theta)=t_{i}\left(\theta^{\prime}\right)$ for all $i \in N$ and use the characterization in Lemma 1 to derive a contradiction.

Consider $\theta^{\prime \prime}$, a 1-profile of $\theta$ such that $\theta_{1}^{\prime \prime}=\theta_{1}^{\prime}$. By efficiency, $\sigma\left(\theta^{\prime \prime}\right)=\sigma(\theta)$ and by strategyproofness, $t_{1}\left(\theta^{\prime \prime}\right)=t_{1}(\theta)$. If $t_{j}\left(\theta^{\prime \prime}\right) \neq t_{j}(\theta)$ for some $j \neq 1$, then the coalition $\{1, j\}$ can manipulate at either $\theta$ or $\theta^{\prime \prime}$. Hence, $t_{i}\left(\theta^{\prime \prime}\right)=$ $t_{i}(\theta)$ for all $i \in N$. Therefore, $g_{i}\left(\theta_{-i}^{\prime \prime}\right)=g_{i}\left(\theta_{-i}\right)$ for all $i \in N$.

Observe that $\theta^{\prime}$ is a 2-profile of $\theta^{\prime \prime}$. Since $\sigma\left(\theta^{\prime}\right)=\sigma(\theta)=\sigma\left(\theta^{\prime \prime}\right)$, strategyproofness implies that $t_{2}\left(\theta^{\prime}\right)=t_{2}\left(\theta^{\prime \prime}\right)$ and using the argument in the previous paragraph, it follows that $t_{i}\left(\theta^{\prime}\right)=t_{i}\left(\theta^{\prime \prime}\right)=t_{i}(\theta)$ for all $i \in N$. This implies that $g_{i}\left(\theta_{-i}^{\prime}\right)=g_{i}\left(\theta_{-i}^{\prime \prime}\right)=g_{i}\left(\theta_{-i}\right)$ for all $i \in N$. Using (1), it follows that $0=\sum_{i \in N}\left(t_{i}\left(\theta^{\prime}\right)-t_{i}(\theta)\right)=-\delta^{\prime}<0$, a contradiction.

In view of the fact that strong group strategyproofness is a demanding requirement - it requires that no group of agents can deviate in a manner where only some benefit strictly - the negative result in Theorem 1 is not surprising. We will now show that positive results can be obtained with weak group strategyproofness. To prove these results, we need a mild technical condition which is given below.

Definition 6. A mechanism $(\sigma, t)$ is continuous if for all $i, j, t_{i}\left(\theta_{j}, \theta_{-j}\right)$ is a continuous function of $\theta_{j}$ except when $\theta_{j}=\theta_{k}$ for some $k \neq j$.

Fixing the announcements of all $k \neq j$, we can regard $t_{i}$ as a function of $j$ 's announcement $\theta_{j}$. Continuity requires that $t_{i}$ varies continuously with $\theta_{j}$ except at profiles where $j$ 's position in the queue changes. In an efficient mechanism, this will occur when $\theta_{j}=\theta_{k}$ for some $k \neq j$.

Definition 7. A mechanism $(\sigma, t)$ is said to be a linearized pivotal mecha$n i s m$ if for all profiles $\theta$, 
(1) $t_{i}(\theta)=-\sum_{j \in P_{i}^{\prime}(\sigma(\theta))} \theta_{j}+\sum_{j \neq i} \alpha_{i j}\left(\theta_{-i}\right) \theta_{j}$ for all $i \in N$,

(2) $\alpha_{i j}\left(\theta_{-i}\right) \in\{0,1\}$ for all $i, j \in N, i \neq j$,

(3) $\alpha_{i j}\left(x, \theta_{-i-j}\right)=\alpha_{j i}\left(x, \theta_{-i-j}\right)$ for all $i, j \in N, i \neq j$ and all $x \in \Re_{+}$.

Remark 3. Comparing the above transfers with (4), it follows that the linearized pivotal mechanisms are a small subset of the set of efficient and strategyproof mechanisms.

Theorem 2. An efficient mechanism $(\sigma, t)$ is weak group strategyproof and continuous only if it is a linearized pivotal mechanism.

Proof: Let $(\sigma, t)$ be an efficient mechanism satisfying weak group strategyproofness and continuity. Let $\theta$ be a profile such that $\theta_{1}>\ldots>\theta_{n}$. By efficiency, $\sigma_{i}(\theta)=i$ for all $i \in N$. By Lemma 2 we know that an efficient mechanism is strategyproof if and only if the transfers are of the form

$$
t_{i}(\theta)=-\sum_{j \in P_{i}^{\prime}(\sigma(\theta))} \theta_{j}+h_{i}\left(\theta_{-i}\right)
$$

We work out the restrictions that weak group strategyproofness and continuity impose on the set of allowable transfers. Let $S=\{i, j\}$ be such that $j=i+1$ and let $\theta^{\prime}$ be a $S$-profile of $\theta$.

Claim 1: Suppose $\sigma_{k}\left(\theta^{\prime}\right)=\sigma_{k}(\theta)$ for all $k \notin S$. Then, $h_{i}\left(\theta_{j}, \theta_{-S}\right)=$ $\alpha_{i}\left(\theta_{j}, \theta_{-S}\right) \theta_{j}+f_{i j}\left(\theta_{-S}\right), \alpha_{i}\left(\theta_{j}, \theta_{-S}\right) \in\{0,1\}$ for $i, j \in S, i \neq j$.

Proof: We first show that continuity implies that there exists $\epsilon>0$ such that

$$
\begin{gathered}
\text { either } h_{i}\left(x, \theta_{-S}\right)-x \text { is a constant in } N\left(\theta_{j}, \epsilon\right) \\
\text { or } h_{j}\left(y, \theta_{-S}\right) \text { is a constant in } N\left(\theta_{i}, \epsilon\right) .
\end{gathered}
$$

To see this, suppose the contrary. Then, we can choose $\theta_{i}^{\prime}, \theta_{j}^{\prime}, \epsilon$ such that $\theta_{i}^{\prime} \in N\left(\theta_{i}, \epsilon\right), \theta_{j}^{\prime} \in N\left(\theta_{j}, \epsilon\right), N\left(\theta_{i}, \epsilon\right) \cap N\left(\theta_{j}, \epsilon\right)=\emptyset$ and $h_{i}\left(\theta_{j}^{\prime}, \theta_{-S}\right)-\theta_{j}^{\prime} \neq$ $h_{i}\left(\theta_{j}, \theta_{-S}\right)-\theta_{j}, h_{j}\left(\theta_{i}^{\prime}, \theta_{-S}\right) \neq h_{j}\left(\theta_{i}, \theta_{-S}\right)$. If $\left[h_{i}\left(\theta_{j}^{\prime}, \theta_{-S}\right)-\theta_{j}^{\prime}\right]-\left[h_{i}\left(\theta_{j}, \theta_{-S}\right)-\right.$ $\left.\theta_{j}\right]$ and $h_{j}\left(\theta_{i}^{\prime}, \theta_{-S}\right)-h_{j}\left(\theta_{i}, \theta_{-S}\right)$ have the same sign, then $S=\{i, j\}$ manipulates at either $\theta$ or $\theta^{\prime}$. Hence, assume without loss of generality that $\left[h_{i}\left(\theta_{j}^{\prime}, \theta_{-S}\right)-\theta_{j}^{\prime}\right]-\left[h_{i}\left(\theta_{j}, \theta_{-S}\right)-\theta_{j}\right]>0$ and $h_{j}\left(\theta_{i}^{\prime}, \theta_{-S}\right)-h_{j}\left(\theta_{i}, \theta_{-S}\right)<0$. Consider the profiles $\theta^{i}=\left(\theta_{i}^{\prime}, \theta_{j}, \theta_{-S}\right)$ and $\theta^{j}=\left(\theta_{i}, \theta_{j}^{\prime}, \theta_{-S}\right)$. It follows from strategyproofness that $t_{i}\left(\theta^{i}\right)=t_{i}(\theta)$ and $t_{j}\left(\theta^{j}\right)=t_{j}(\theta)$. However, since $t_{j}\left(\theta^{i}\right)<t_{j}(\theta)$ and $t_{i}\left(\theta^{j}\right)>t_{i}(\theta)$ it follows that $S$ can profitably manipulate at $\theta^{i}$ via $\theta^{j}$. This contradiction establishes that either (6) or (7) holds.

Next, consider the $S$-profile $\theta^{\prime \prime}$ of $\theta$ such that $\theta_{i}^{\prime \prime}=\theta_{j}, \theta_{j}^{\prime \prime}=\theta_{i}$. It is easy to see that $\sigma_{k}\left(\theta^{\prime \prime}\right)=\sigma_{k}(\theta)$ for all $k \notin S, \sigma_{i}\left(\theta^{\prime \prime}\right)=j, \sigma_{j}\left(\theta^{\prime \prime}\right)=i$. Using the same logic as before, it follows that there exists $\epsilon>0$ such that either $h_{j}\left(x, \theta_{-S}\right)-x$ is a constant in $N\left(\theta_{j}, \epsilon\right)$ or $h_{i}\left(y, \theta_{-S}\right)$ is a constant in $N\left(\theta_{j}, \epsilon\right)$. 
Suppose that (6) holds. If there is no $\epsilon$ such that $h_{j}\left(x, \theta_{-S}\right)-x$ is a constant in $N\left(\theta_{j}, \epsilon\right)$, then it must be the case that $h_{i}\left(x, \theta_{-S}\right)$ is a constant in some $N\left(\theta_{i}, \epsilon^{\prime}\right)$. Consider the $i$-profile $\tilde{\theta}$ of $\theta$ such that $\tilde{\theta}_{i} \in N\left(\theta_{j}, \epsilon\right)$ and $\theta_{i}>\theta_{j}$. By strategyproofness, the transfer $h_{i}\left(\theta_{j}, \theta_{-S}\right)$ remains unchanged since $\sigma_{i}(\tilde{\theta})=\sigma_{i}(\theta)$. We have a contradiction since both $h_{i}\left(x, \theta_{-S}\right)-x$ and $h_{i}\left(x, \theta_{-S}\right)$ are constant for $x \in N\left(\theta_{j}, \epsilon\right) \cap N\left(\theta_{i}, \epsilon^{\prime}\right)$. Hence, if $h_{i}\left(x, \theta_{-S}\right)-$ $x$ is a constant in some $N\left(\theta_{j}, \epsilon^{\prime}\right)$ then there exists $\epsilon>0$ such that both $h_{i}\left(x, \theta_{-S}\right)-x$ and $h_{j}\left(x, \theta_{-S}\right)$ are constants in $N\left(\theta_{j}, \epsilon\right)$.

Alternatively, suppose (7) holds. If there is no $\epsilon$ such that $h_{i}\left(y, \theta_{-S}\right)$ is a constant in $N\left(\theta_{j}, \epsilon\right)$, then it must be true that $h_{j}\left(x, \theta_{-S}\right)-x$ is a constant in some $N\left(\theta_{j}, \epsilon^{\prime}\right)$. By taking $\bar{\theta}$, a $j$-profile of $\theta$ such that $\bar{\theta}_{j}<\theta_{i}$ and $\bar{\theta}_{j} \in N\left(\theta_{i}, \epsilon\right) \cap N\left(\theta_{j}, \epsilon^{\prime}\right)$, we can get a similar contradiction as above. Hence, it must be true that if (7) holds, then $h_{i}\left(y, \theta_{-S}\right)$ is a constant in some $N\left(\theta_{j}, \epsilon\right)$.

We have thus shown that: if $\theta_{i}>\theta_{j}$ and $\sigma_{i}(\theta)-\sigma_{j}(\theta)=1$ then there exists $N\left(\theta_{j}, \epsilon\right)$ where either $h_{i}\left(y, \theta_{-S}\right)-y$ and $h_{j}\left(y, \theta_{-S}\right)-y$ are both constants or $h_{i}\left(y, \theta_{-S}\right)$ and $h_{j}\left(y, \theta_{-S}\right)$ are both constants. Therefore, fixing $\theta_{-S}$, the functions $h_{i}$ and $h_{j}$ can be written as

$$
h_{k}\left(y, \theta_{-S}\right)=\alpha_{k l}\left(y, \theta_{-S}\right) y+f_{k l}\left(\theta_{-S}\right), \quad \alpha_{k l}\left(y, \theta_{-S}\right) \in\{0,1\}, k \neq l .
$$

Note that since the $h_{i}$ functions are continuous, $\alpha_{i j}\left(y, \theta_{-S}\right)$ is constant for all profiles $\theta_{-i}, \theta_{-i}^{\prime}$ such that $\sigma_{k}\left(\theta_{-i}\right)=\sigma_{k}\left(\theta_{-i}^{\prime}\right)$ for all $k \in N \backslash\{i\}$.

We extend the argument by considering $j$ and her successor (say $k$ ). It follows from the above that

$$
h_{l}\left(z, \theta_{-j-k}\right)=\alpha_{l m}\left(z, \theta_{-j-k}\right) z+f_{l m}\left(\theta_{-j-k}\right), l, m=j, k, l \neq m, \alpha_{l m} \in\{0,1\} .
$$

Equating the two expressions for $h_{j}$, we get

$$
\alpha_{j i}\left(\theta_{i}, \theta_{-i-j}\right) \theta_{i}+f_{j i}\left(\theta_{-i-j}\right)=\alpha_{j k}\left(\theta_{k}, \theta_{-j-k}\right) \theta_{k}+f_{j k}\left(\theta_{-j-k}\right)
$$

Noting that the $\alpha_{i j}$ functions only take on the values zero or one, we can equate the terms on either side to conclude that

$$
h_{j}\left(\theta_{i}, \theta_{k}, \theta_{-i-j-k}\right)=\alpha_{j i}\left(\theta_{i}, \theta_{-i-j}\right) \theta_{i}+\alpha_{j k}\left(\theta_{k}, \theta_{-j-k}\right) \theta_{k}+f_{j}\left(\theta_{-i-j-k}\right)
$$

Consider the profile $\theta^{\prime}$ where $\theta_{i}^{\prime}=\theta_{j}, \theta_{j}^{\prime}=\theta_{i}$ and $\theta_{l}^{\prime}=\theta_{l}$ for all $l \neq i, j$. It is straightforward to verify that $\sigma_{i}\left(\theta^{\prime}\right)=j, \sigma_{j}\left(\theta^{\prime}\right)=i$ and $\sigma_{l}\left(\theta^{\prime}\right)=\sigma_{l}(\theta)$ for all $l \neq i, j$. It follows that in the profile $\theta^{\prime}, i$ and $k$ are neighbours. Using the previous arguments, it follows that

$$
h_{i}\left(\theta_{k}, \theta_{-i-k}\right)=\alpha_{i k}\left(\theta_{-i}\right) \theta_{k}+f_{i k}\left(\theta_{-i-k}\right)
$$


Comparing (8) and (12) and noting again that the $\alpha_{i j}$ functions are binary, it follows that

$$
h_{i}\left(\theta_{j}, \theta_{k}, \theta_{-i-j-k}\right)=\alpha_{i j}\left(\theta_{-i}\right) \theta_{j}+\alpha_{i k}\left(\theta_{-i}\right) \theta_{k}+f_{i}\left(\theta_{-i-j-k}\right)
$$

We use the above arguments recursively to compare $i$ and $j$ with all other agents. It follows therefore that the functions $h_{i}$ must be of the form

$$
h_{i}\left(\theta_{-i}\right)=\sum_{j \neq i} \alpha_{i j}\left(\theta_{-i}\right) \theta_{j}, \alpha_{i j} \in\{0,1\}, i \neq j, i \in N .
$$

This completes the proof.

Observe that to derive the necessity of the linearized pivotal mechanism, we have just considered two player deviations. However, the implication of our necessity result is significant and is captured in the next two theorems.

Theorem 3. If $n=3$, then there exists an efficient mechanism satisfying weak group strategyproofness and budget balance.

Proof: Consider the mechanism which at any profile $\theta$ selects the efficient order and implements the following transfers:

$$
t_{i}(\theta)=\left\{\begin{array}{cl}
-\theta_{j} & \text { if } \sigma_{i}(\theta)=1, \sigma_{j}(\theta)=2, \\
0 & \text { if } \sigma_{i}(\theta)=2, \\
\theta_{j} & \text { if } \sigma_{i}(\theta)=3, \sigma_{j}(\theta)=2 .
\end{array}\right.
$$

The transfers have been derived from the linearized pivotal mechanism by setting

$$
\alpha_{i j}\left(\theta_{j}, \theta_{k}\right)= \begin{cases}1 & \text { if } \sigma_{j}\left(\theta_{j}, \theta_{k}\right)=2 \\ 0 & \text { otherwise }\end{cases}
$$

for all distinct $i, j, k$. This mechanism is strategyproof and budget balanced (see Mitra [6]), so we only need to show that it cannot be manipulated by a coalition of size 2 or 3 . Efficiency and budget balance implies that the mechanism is Pareto optimal and cannot be manipulated by the grand coalition $N=\{1,2,3\}$. Therefore, it suffices to consider coalitions of size 2 .

Without loss of generality, suppose that $\theta_{1} \geq \theta_{2} \geq \theta_{3}$. Using our efficient rule we get $\sigma_{i}(\theta)=i$ for all $i$. Suppose that a coalition $S$ of size 2 can manipulate at $\theta$ through $\theta^{\prime}$, a $S$-profile of $\theta$. We claim that $\sigma_{i}\left(\theta^{\prime}\right) \neq 2$ for any $i \in S$. To see this, suppose $\sigma_{i}\left(\theta^{\prime}\right)=2$. If $\sigma_{i}(\theta)=1$, then $i$ 's gain from the deviation is $\theta_{2}-\theta_{1} \leq 0$ and hence she does not benefit strictly. If $\sigma_{i}(\theta)=3$, then the gain is $\theta_{3}-\theta_{2} \leq 0$. And lastly if $\sigma_{i}(\theta)=2$, then $i$ 's benefit from deviation is zero.

Suppose $S=\{1,3\}$. If $\sigma_{i}\left(\theta^{\prime}\right)=\sigma_{i}(\theta)$ for all $i \in S$, then it is easy to verify that $u_{1}\left(\sigma(\theta), t(\theta), \theta_{1}\right)=u_{1}\left(\sigma\left(\theta^{\prime}\right), t\left(\theta^{\prime}\right), \theta_{1}\right)=-\theta_{2}, u_{3}\left(\sigma(\theta), t(\theta), \theta_{1}\right)=$ 
$u_{3}\left(\sigma\left(\theta^{\prime}\right), t\left(\theta^{\prime}\right), \theta_{3}\right)=\theta_{2}-2 \theta_{3}$ and hence neither agent gains from the deviation. Hence, $\sigma_{i}\left(\theta^{\prime}\right) \neq \sigma_{i}(\theta)$ for some $i \in S$. Since $\sigma_{i}\left(\theta^{\prime}\right) \neq 2$ for all $i \in S$, it follows that $\sigma_{1}\left(\theta^{\prime}\right)=3, \sigma_{3}\left(\theta^{\prime}\right)=1$. Agent 1's gain from the deviation is $\theta_{2}-2 \theta_{1}+\theta_{1} \leq 0$ since $\theta_{1} \geq \theta_{2}$. This is a contradiction since we have assumed that $S$ can manipulate at $\theta$ by reporting $\theta^{\prime}$.

Suppose $S=\{1,2\}$. If $\sigma_{2}\left(\theta^{\prime}\right)=3$, then her gain in utility is $\theta_{3}-2 \theta_{2}+\theta_{2}=$ $\theta_{3}-\theta_{2} \leq 0$ which shows that 2 does not gain from the deviation. Hence, using $\sigma_{i}\left(\theta^{\prime}\right) \neq 2$ for all $i \in S$, we must have $\sigma_{2}\left(\theta^{\prime}\right)=1, \sigma_{1}\left(\theta^{\prime}\right)=3$. Agent 1 's gain in utility is $\theta_{3}-2 \theta_{1}+\theta_{2} \leq 0$ since $\theta_{1} \geq \theta_{2} \geq \theta_{3}$. So, we have a contradiction again.

Finally, suppose $S=\{2,3\}$. If $\sigma_{2}\left(\theta^{\prime}\right)=1$, then 2's gain from the deviation is $-\theta_{1}+\theta_{2} \leq 0$. So, we must have $\sigma_{2}\left(\theta^{\prime}\right)=3, \sigma_{3}\left(\theta^{\prime}\right)=1$. Agent 3's gain from the deviation is $-\theta_{1}-\theta_{2}+2 \theta_{3} \leq 0$. Hence, we again have a contradiction. This completes the proof that the above mechanism is group strategyproof.

It turns out that weak group strategyproofness, efficiency and budget balance are compatible only when there are three agents. The following result will be used to establish the negative result when $n \neq 3$.

Proposition 1. Suppose $n>2$. An efficient mechanism $(\sigma, t)$ is strategyproof and budget balanced if and only if

$$
t_{i}(\theta)=\sum_{j \in P_{i}(\sigma(\theta))}\left(\frac{\sigma_{j}(\theta)-1}{n-2}\right) \theta_{j}-\sum_{j \in P_{i}^{\prime}(\sigma(\theta))}\left(\frac{n-\sigma_{j}(\theta)}{n-2}\right) \theta_{j}+\gamma_{i}\left(\theta_{-i}\right)
$$

where $\sum_{i \in N} \gamma_{i}\left(\theta_{-i}\right)=0$.

Proof: In condition (4) of Lemma 2 we substitute

$$
h_{i}\left(\theta_{-i}\right)=\sum_{j \neq i}\left(\frac{\sigma_{j}\left(\theta_{-i}\right)-1}{n-2}\right) \theta_{j}+\gamma_{i}\left(\theta_{-i}\right) .
$$

Using this substitution we get that an efficient mechanism is strategyproof if and only if the transfer satisfies (15). Condition (15) is obtained by using the following result in (16) which is a consequence of efficiency of the mechanism.

$$
\sigma_{j}\left(\theta_{-i}\right)= \begin{cases}\sigma_{j}(\theta) & \text { if } \sigma_{j}(\theta)<\sigma_{i}(\theta) \\ \sigma_{j}(\theta)-1 & \text { if } \sigma_{j}(\theta)>\sigma_{i}(\theta)\end{cases}
$$

It follows that the transfer given by (15) has the property that $\sum_{i \in N} t_{i}(\theta)=$ $\sum_{i \in N} \gamma_{i}\left(\theta_{-i}\right) .{ }^{6}$ If we add budget balance to $(15)$, the result follows.

Theorem 4. If $n \neq 3$, there are no efficient mechanisms satisfying continuity, weak group strategyproofness and budget balance.

\footnotetext{
${ }^{6}$ See Mitra [6].
} 
Proof: Let $(\sigma, t)$ be a mechanism satisfying all the requirements in the hypothesis. Suppose $n=2$. Let $\theta$ be a profile such that $\theta_{1}>\theta_{2}$. By efficiency, $\sigma_{i}(\theta)=i$ for all $i$. Using $(1), t_{1}(\theta)=-\theta_{2}+h_{1}\left(\theta_{2}\right)$ and $t_{2}(\theta)=$ $h_{2}\left(\theta_{1}\right)$. We observe that $h_{1}$ and $h_{2}$ do not depend on the positions of 1 and 2 in the queue. Consider the 1-profile $\theta^{\prime}$ of $\theta$ such that $\theta_{1}^{\prime}<\theta_{2}$. By efficiency, $\sigma_{i}\left(\theta^{\prime}\right)=3-i, i=1,2$. Again, using (1), the transfers are $t_{1}\left(\theta^{\prime}\right)=$ $h_{1}\left(\theta_{2}\right), t_{2}\left(\theta^{\prime}\right)=-\theta_{1}^{\prime}+h_{2}\left(\theta_{1}^{\prime}\right)$. By budget balance at $\theta$ and $\theta^{\prime}$, we have $h_{1}\left(\theta_{2}\right)+h_{2}\left(\theta_{1}\right)-\theta_{2}=0$ and $h_{1}\left(\theta_{2}\right)+h_{2}\left(\theta_{1}^{\prime}\right)-\theta_{1}^{\prime}=0$. Hence, $h_{2}\left(\theta_{1}^{\prime}\right)-h_{2}\left(\theta_{1}\right)=$ $\theta_{1}^{\prime}-\theta_{2}$. We have a contradiction because the left hand side is independent of $\theta_{2}$ while the right hand side is a function of $\theta_{2}$.

Suppose that $n=4$. Let $\theta$ be a profile such that $\theta_{1}>\theta_{2}>\theta_{3}>\theta_{4}$. Then, using the necessary condition for efficiency, continuity and weak group strategyproofness in Theorem 2, we have

$$
t_{1}(\theta)=-\theta_{2}-\theta_{3}-\theta_{4}+\sum_{j=2}^{4} \alpha_{1 j}\left(\theta_{-1}\right) \theta_{j}
$$

where $\alpha_{1 j} \in\{0,1\}$ for all $j \neq 1$. Using the condition for strategyproofness, efficiency and budget balance in (15), it also follows that

$$
t_{1}(\theta)=-\theta_{2}-\frac{\theta_{3}}{2}+\sum_{j=2}^{4} \beta_{1 j}\left(\theta_{-1}\right) \theta_{j}
$$

Hence comparing the two expressions above and using the fact that the mechanism is continuous, it follows that

$$
\alpha_{14}\left(\tilde{\theta}, \theta_{2}, \theta_{3}\right)-\beta_{14}\left(\tilde{\theta}, \theta_{2}, \theta_{3}\right)=1
$$

for all $\tilde{\theta}<\theta_{3}$. Consider another profile $\theta^{\prime}$ such that $\theta_{i}^{\prime}=\theta_{i}$ for $i=1,2$, $\theta_{3}^{\prime}=\theta_{4}, \theta_{4}^{\prime}=\theta_{3}$. Note that the efficient queue at $\theta^{\prime}$ involves players 3 and 4 exchanging positions. Using the two different expressions for $t_{1}$ at $\theta^{\prime}$, it follows that

$$
\alpha_{14}\left(\tilde{\theta}, \theta_{2}, \theta_{4}\right)-\beta_{14}\left(\tilde{\theta}, \theta_{2}, \theta_{4}\right)=\frac{1}{2}
$$

for all $\theta_{4}<\tilde{\theta}<\theta_{3}$. The above two expressions gives a contradiction at any profile $\bar{\theta}$ such that $\bar{\theta}_{i}=\theta_{i}$ for $i=1,2,3$ and $\theta_{4}<\bar{\theta}_{4}<\theta_{3}$.

The general case follows by considering a profile $\theta$ such that $\theta_{1}>\theta_{2}>$ $\theta_{3}>\theta_{4}$ and $\theta_{j}<\theta_{4}$ for all $j \neq 1,2,3,4$.

Definition 8. A mechanism $(\sigma, t)$ is a $k$-pivotal mechanism if it is a linearized pivotal mechanism and there exists $k \in\{1, \ldots, n\}$ such that for all profiles $\theta$,

$$
\alpha_{i j}\left(\theta_{-i}\right)= \begin{cases}1 & \text { if } \sigma_{j}\left(\theta_{-i}\right) \geq k \\ 0 & \text { otherwise }\end{cases}
$$


Observation: For all $i \in N$ and for all $\theta \in \Re_{+}^{n}$, the transfers in the $k$-pivotal mechanism have the following form:

$$
t_{i}(\theta)=\left\{\begin{array}{cc}
-\sum_{j \in X_{1}(i, k ; \theta)} \theta_{j} & \text { if } \sigma_{i}(\theta)<k \\
0 & \text { if } \sigma_{i}(\theta)=k \\
\sum_{j \in X_{2}(i, k ; \theta)} \theta_{j} & \text { if } \sigma_{i}(\theta)>k
\end{array}\right.
$$

where $X_{1}(i, k ; \theta)=\left[q \in N \mid \sigma_{q}(\theta) \in\left\{\sigma_{i}(\theta)+1, \ldots, k\right\}\right]$ and $X_{2}(i, k ; \theta)=$ $\left[q \in N \mid \sigma_{q}(\theta) \in\left\{k, \ldots, \sigma_{i}(\theta)-1\right\}\right]$. An $n$-pivotal mechanism is the standard pivotal mechanism given by condition 3 .

Theorem 5. An efficient $k$-pivotal mechanism is weak group strategyproof.

Proof: Let $\theta$ be a profile and without loss of generality, assume that $\sigma_{i}(\theta)=$ $i$ for all $i \in N$. Note that efficiency implies that $\theta_{1} \geq \ldots \geq \theta_{n}$. We show that there does not exist a profitable deviation for any coalition $S \subset N$.

Claim 1: Let $S \subset N$ deviate at $\theta$ through the $S$-profile $\theta^{\prime}$. Then, $\sigma_{i}\left(\theta^{\prime}\right) \neq k$ for all $i \in S$.

Proof: Suppose $\sigma_{i}\left(\theta^{\prime}\right)=k$ for $i \in S$. If $\sigma_{i}(\theta)=k$, then $t_{i}(\theta)=t_{i}\left(\theta^{\prime}\right)=0$ and hence, $i$ does not benefit strictly from the deviation. Suppose $\sigma_{i}(\theta)=i<k$. Then, the benefit to player $i$ from the deviation is $\sum_{l=i+1}^{k} \theta_{l}-(k-i) \theta_{i} \leq 0$ because $\theta_{i} \geq \theta_{l}$ for all $l \geq i+1$. Lastly, suppose $\sigma_{i}(\theta)=i>k$. The maximum benefit to player $i$ from the deviation is $(i-k) \theta_{i}-\sum_{l=k}^{i-1} \theta_{l} \leq 0$ because $\theta_{i} \leq \theta_{l}$ for $l<i$. Hence, in all cases, the player moving to position $k$ does not benefit from the deviation. This implies that $S \neq N$ and there exists $i \notin S$. In particular, if $\sigma_{i}\left(\theta^{\prime}\right)=k$, then $i \notin S$.

Claim 2: Let $\sigma_{i}\left(\theta^{\prime}\right)=k$. Then, for all $j \in S$, if $\sigma_{j}(\theta)>i$, then $\sigma_{j}\left(\theta^{\prime}\right)>k$ and if $\sigma_{j}(\theta)<i$ then $\sigma_{j}\left(\theta^{\prime}\right)<k$.

Proof: Suppose $\sigma_{j}(\theta)=j>i=\sigma_{i}(\theta)$ and $\sigma_{j}\left(\theta^{\prime}\right)=j^{\prime}<k$. Then, $j$ 's maximal profit from the deviation is $\Delta u_{j}=\left(j-j^{\prime}\right) \theta_{j}-\sum_{l=k}^{j-1} \theta_{l}-\left(k-j^{\prime}\right) \theta_{i} \leq$ 0 . Conversely, suppose $\sigma_{j}(\theta)=j<i=\sigma_{i}(\theta)$ and $\sigma_{j}\left(\theta^{\prime}\right)=j^{\prime}>k$. In this case, $j$ 's maximal gain from the deviation is $\Delta u_{j}=-\left(j^{\prime}-j\right) \theta_{j}+\sum_{l=j+1}^{k} \theta_{l}+$ $\left(j^{\prime}-k\right) \theta_{k} \leq 0$.

Claim 2 implies that if $\sigma_{i}\left(\theta^{\prime}\right)=k$ then $\sigma_{i}(\theta)=k$ : indeed, since members of the deviating coalition preserve their positions with respect to $i$ and all non-deviating members preserve their positions with respect to each other, we would get a contradiction if $\sigma_{i}(\theta) \neq k$. Thus, the only possible deviations are ones where deviating members preserve their positions vis-a-vis $k$ who herself is not a member of the deviating coalition.

Let $S=S_{1} \cup S_{2}$ where $S_{1}=\left\{i \in S \mid \sigma_{i}(\theta)<k\right\}$ and $S_{2}=\left\{i \in S \mid \sigma_{i}(\theta)>\right.$ $k\}$. Since the transfers of agents in $S_{1}$ are not affected by the waiting costs of agents in $S_{2}$ and vice versa and since the deviation by $S$ does not 
involve agents changing their positions relative to $k$, we can without loss of generality, let $S_{1} \cap S_{2}=\emptyset$.

Suppose $S_{1} \neq \emptyset$. If there exists $i \in S_{1}$ such that $\sigma_{i}(\theta)<\sigma_{i}\left(\theta^{\prime}\right)$, then $i$ 's gain from the deviation is $\Delta u_{i}=-\left(\sigma_{i}\left(\theta^{\prime}\right)-\sigma_{i}(\theta)\right) \theta_{i}+\sum_{l=\sigma_{i}(\theta)+1}^{\sigma_{i}\left(\theta^{\prime}\right)} \theta_{l} \leq 0$. Suppose $\sigma_{i}(\theta)=\sigma_{i}\left(\theta^{\prime}\right)$ for all $i \in S_{1}$. Then, in fact $\left.\sigma(\theta)=\sigma_{(} \theta^{\prime}\right)$. Let $j=\operatorname{argmin}_{i \in S_{1}} \sigma_{i}\left(\theta_{i}\right)$. Then, $t_{j}\left(\theta^{\prime}\right)=t_{j}(\theta)$ and hence $j$ does not benefit from the deviation. Finally, suppose $\sigma_{j}\left(\theta^{\prime}\right) \leq \sigma_{j}(\theta)$ for all $j \in S_{1}$ with at least one strict inequality. In this case, we must in fact have $\sigma_{j}\left(\theta^{\prime}\right)<\sigma_{j}(\theta)$ for all $j \in S_{1}$ : otherwise, the agents whose queue positions are unchanged do not benefit. Furthermore, $\theta_{j}^{\prime} \geq \theta_{j}$ for all $j \in S_{1}$ : this follows from efficiency. (In order to move ahead of a non-deviating member $i, j$ 's announcement has to be higher than $\theta_{i}$.) Since the transfers require that $i$ pays $\theta_{j}$ if she moves ahead of $j$ in the queue, it follows that no $i \in S_{1}$ can be better off.

Suppose $S_{2} \neq \emptyset$. If there exists $i \in S_{2}$ such that $\sigma_{i}(\theta)>\sigma_{i}\left(\theta^{\prime}\right)$, then $i$ 's maximal benefit from the deviation is $\Delta u_{i}=\left(\sigma_{i}(\theta)-\sigma_{i}\left(\theta^{\prime}\right)\right) \theta_{i}-\sum_{l=\sigma_{i}\left(\theta^{\prime}\right)}^{\sigma_{i}(\theta)-1} \theta_{l} \leq$ 0 because $\theta_{i} \leq \theta_{l}$ for all $l<i$. Suppose $\sigma_{i}(\theta)=\sigma_{i}\left(\theta^{\prime}\right)$ for all $i \in S_{2}$. Then, in fact $\sigma(\theta)=\sigma\left(\theta^{\prime}\right)$. Let $j=\operatorname{argmax}_{i \in S_{2}} \sigma_{i}\left(\theta_{i}\right)$. Then, $t_{j}\left(\theta^{\prime}\right)=t_{j}(\theta)$ and hence $j$ does not benefit from the deviation. Finally, suppose $\sigma_{j}\left(\theta^{\prime}\right) \geq \sigma_{j}(\theta)$ for all $j \in S_{2}$ with at least one strict inequality. Then, we must have $\sigma_{j}\left(\theta^{\prime}\right)>\sigma_{j}(\theta)$ for all $j \in S_{2}$ : otherwise, the agents whose queue positions are unchanged do not benefit. Furthermore, $\theta_{j}^{\prime} \leq \theta_{j}$ for all $j \in S_{2}$ : this follows from efficiency. Since the transfers require that $i$ receives $\theta_{j}$ if she moves behind $j$ in the queue, it follows that the benefits of moving behind $\left(\theta_{j}\right)$ are less than the $\operatorname{cost} \theta_{i}$.

Let $[x]_{+}$be the smallest integer value of $x$ greater than or equal to $x$.

Proposition 2. An efficient $k$-pivotal mechanism is feasible if and only if $k \geq\left[\frac{n+1}{2}\right]_{+}$.

Proof: From the definition of $k$-pivotal mechanism it follows that

$$
\sum_{i \in N} t_{i}(\theta)=-\sum_{j \in Y_{1}(k ; \theta)}\left(\sigma_{j}(\theta)-1\right) \theta_{j}+\sum_{j \in Y_{2}(k ; \theta)}\left(n-\sigma_{j}(\theta)\right) \theta_{j}
$$

where $Y_{1}(k ; \theta)=\left\{j \in N \mid \sigma_{j}(\theta) \leq k\right\}$ and $Y_{2}(k ; \theta)=\left\{j \in N \mid \sigma_{j}(\theta) \geq k\right\}$. Consider a state $\theta$ such that $\theta_{1}=\ldots=\theta_{n}=x>0$. Using (21) we get

$$
\sum_{i \in N} t_{i}(\theta)=\left[-\sum_{p=1}^{k}(p-1)+\sum_{q=k}^{n}(n-q)\right] x=\frac{n(n+1-2 k) x}{2}
$$

For feasibility it is necessary that $\frac{n(n+1-2 k) x}{2} \leq 0$ which implies that $k \geq \frac{n+1}{2}$. This proves the necessity of feasibility. 
For sufficiency consider the $k$-th pivotal mechanism with $k \geq\left[\frac{n+1}{2}\right]_{+}$. Consider any profile $\theta$ where $\theta_{(1)} \geq \ldots \geq \theta_{(n)} \geq 0$ and $\theta_{(p)}$ is the $p$-th highest waiting cost for $p \in\{1, \ldots, n\}$. If $k>\frac{n+1}{2}$, then from (21) we get

$\sum_{i \in N} t_{i}(\theta)=-\sum_{p=1}^{n+1-k}(p-1)\left(\theta_{(p)}-\theta_{(n+1-p)}\right)-\sum_{q=n+1-k}^{k-1}(q-1) \theta_{(q)}-[2 k-(n+1)] \theta_{(k)}$

Since $\theta_{(p)} \geq \theta_{(n+1-p)}$ for all $p \in\{1, \ldots, n+1-k\}, \theta_{(n)} \geq 0$ and $2 k>(n+1)$ we get $\sum_{i \in N} t_{i}(\theta) \leq 0$. If $k=\frac{n+1}{2}$, then from (21) we get

$$
\sum_{i \in N} t_{i}(\theta)=-\sum_{p=1}^{n+1-k}(p-1)\left(\theta_{(p)}-\theta_{(n+1-p)}\right)
$$

Since $\theta_{(p)} \geq \theta_{(n+1-p)}$ for all $p \in\{1, \ldots, n+1-k\}$ we get $\sum_{i \in N} t_{i}(\theta) \leq 0$ and the prove is complete.

Remark 4. If $n=3$, then Proposition 2 shows that we can get efficiency, weak group strategyproofness and budget balance if and only if $k=2$. Thus, the mechanism identified in Theorem 3 is essentially unique.

\section{Conclusion}

We have shown that, in general, group strategyproofness, efficiency and budget balance cannot be satisfied jointly in the single server queueing model. If we weaken budget balance to feasibility, then it is possible to obtain positive results so long as we use the weak variant of group strategyproofness. The main contribution of the paper has been to obtain necessary and sufficient conditions for weak group strategyproofness and efficiency. We note that the necessity condition has been obtained by considering only two player deviations and hence the 'gap' between our necessity and sufficiency results is quite 'thin.'

We have then used our sufficiency condition to identify the subclass of mechanisms which are feasible. It is quite easy to verify that the $k$-pivotal mechanism where $k=\left[\frac{n+1}{2}\right]_{+}$is the 'best' feasible mechanism from a welfare point of view since it minimizes the monetary loss under all possible profiles. We have also observed that for $n=3$, the 'best' feasible mechanism is also budget balanced and, given our necessity result, it is also unique.

One thing that we have not done is to examine the possibility of satisfying individual rationality which would guarantee non-negative utility to all agents under all profiles. It is easy to verify that the 1-pivotal mechanism is compatible with efficiency, weak group strategyproofness and individual rationality. Our guess is that, given our necessity result, the 1-pivotal 
mechanism is the only mechanism that satisfies efficiency, weak group strategyproofness and individual rationality. However, we did not pursue this line of research since the 1-pivotal mechanism is not feasible.

One interesting and challenging open problem would be to extend our analysis to the multiple servers case.

\section{REFERENCES}

[1] Bogomolnaia, A., Moulin, H., 2004. Random matching under dichotomous preferences. Econometrica 72, 257-279.

[2] Clarke, E.H., 1971. Multi-part pricing of public goods. Public Choice 11, 17-33.

[3] Groves, T., 1973. Incentives in teams. Econometrica 41, 617-631.

[4] Holmström, B., 1979. Groves schemes on restricted domains. Econometrica 47, 11371144.

[5] Maniquet, F. 2003. A characterization of the Shapley value in queueing problems. Journal of Economic Theory 109, 90-103.

[6] Mitra, M., 2001. Mechanism design in queueing problems. Economic Theory 17, 277305.

[7] Mitra, M., 2002. Achieving the first best in sequencing problems. Review of Economic Design 7, 75-91.

[8] Mitra, M., 2005. Incomplete information and multiple machine queueing problems. European Journal of Operational Research 165, 251-266.

[9] Moulin, H. 1986. Characterizations of the pivotal mechanism. Journal of Public Economics $31,53-78$.

[10] Moulin, H., Shenker, S. 2001. Strategyproof sharing of submodular costs: budget balance versus eficiency. Economic Theory 18, 511533.

[11] Mutuswami, S., 2005. Strategyproofness, non-bossiness and group strategyproofness in a cost sharing model. Economics Letters 89, 83-88.

[12] Suijs, J., 1996. On incentive compatibility and budget balancedness in public decision making. Economic Design 2, 193-209.

[13] Vickrey, W., 1961. Counterspeculation, auctions and competitive sealed tenders. Journal of Finance 16, 8-37.

Economic Research Unit, Indian Statistical Institute, Kolkata

E-mail address: mmitra@isical.ac.in

Department of Economics, University of Essex, Colchester

E-mail address: smutus@essex.ac.uk 